\title{
Identification of Biosimilar for Trimethoprim - Andrographis paniculata Phytochemicals Inhibits Dihydrofolate Reductase (DHFR)
}

\author{
Saranya Shivashankar ${ }^{1}$ (D), Sangeetha Marimuthu Kannan ${ }^{1, *}$ (D) \\ 1 B.S.Abdur Rahman Crescent Institute of Science and Technology, Chennai, India \\ * Correspondence: geethabct@gmail.com; sangeetha@crescent.education;
}

Scopus Author ID 56459964800

Received: 20.01.2021; Revised: 25.02.2021; Accepted: 2.03.2021; Published: 7.03.2021

\begin{abstract}
Staphylococcus aureus a pathogenic bacterium responsible for hospital and communityacquired infections. Trimethoprim is generally administrated for treating S.aureus infection in combination with sulfamethoxazole. But increasing antimicrobial resistance towards antibiotics is a major concern. Trimethoprim targets Dihydrofolate reductase (DHFR), a crucial enzyme involved in nucleic acid and amino acid biosynthesis pathways. DHFR catalyzes the conversion of dihydrofolate to tetrahydrofolate using NADH as a cofactor. Andrographis paniculata is a traditionally used medicinal plant for treating various ailments, including microbial infections. More than 25 bioactive phytochemicals have been reported to exhibit various activities. The aim of the present study is to identify the lead phytochemical(s) mediating antimicrobial property of $A$. paniculata by using computational analysis. Molecular docking of A.paniculata phytochemicals with wild and mutated DHFR were performed. Results reveal phytochemicals interact and exhibit strong binding affinity with active site residues of wild and mutated strains. 14-deoxy-11-oxoandrographolide showed binding energy greater than $10 \mathrm{kCal} / \mathrm{mol}$ with both strains. Further analysis of A. paniculata phytochemicals for their efficacy would lead to the development of potential drugs for the treatment of microbial infections.
\end{abstract}

Keywords: Andrographis paniculata; molecular docking; Staphylococcus aureus.

(C) 2021 by the authors. This article is an open-access article distributed under the terms and conditions of the Creative Commons Attribution (CC BY) license (https://creativecommons.org/licenses/by/4.0/).

\section{Introduction}

Staphylococcus aureus, a commensal bacterium and human pathogen causes a wide range of diseases. S. aureus infections are mainly hospital and community-acquired. It is a major cause of bacteremia, infective endocarditis, skin and soft tissue infections, osteoarticular infections, prosthetic infections, pleuropulmonary infections, and various Staphylococcal clinical syndromes (epidural abscess, meningitis, toxic shock syndrome, urinary tract infection, and septic thrombophlebitis). S. aureus causes common but severe clinical infections [1-3]. S.aureus infections represent a curative challenge as they are associated with mortality and morbidity significantly [4]. Nearly $30 \%$ of human populations are asymptomatic nasal carriers of $S$. aureus [5]. Carriers of S. aureus are at elevated risk of infection and are assumed to be the main source for spreading $S$. aureus strains among individuals. Transmission of $S$. aureus occurs by direct contact, mostly skin-to-skin contact with infected individuals, and also contaminated objects and surfaces play a role [6]. Antimicrobial resistance (AMR) of bacteria from both community and nosocomial origin is emerging as a serious threat to mankind [7]. S.aureus categorized under priority list 2 as highly important bacteria according to World 
Health Organization on the basis of drug resistance and need for antibiotics [8]. Trimethoprim (TMP) is usually administrated for treating $S$. aureus infection as Co-trimoxazole, a combination of trimethoprim and sulfamethoxazole. Increasing antimicrobial resistance of $S$. aureus is a major concern and its continuous change in the clinical disease spectrum [1].

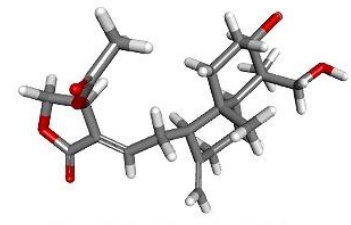

14-acetylandrographolide 14-deoxy-11,12 didehydroandrographolide (73353957)

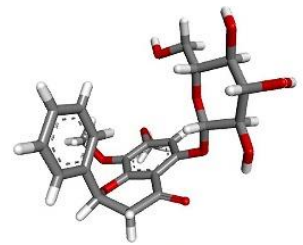

Andrographidine A (13963762)

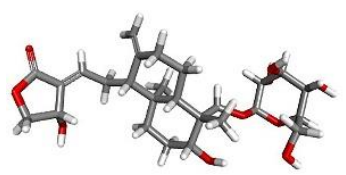

Andrographiside (44593583)

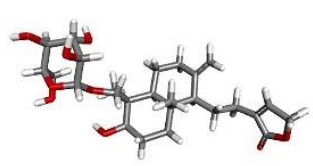

Andropanoside (44575270)

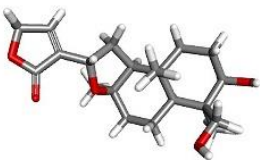

Isoandrographolide (101563021)

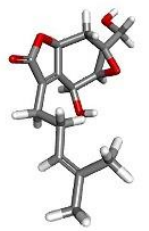

Paniculide B (101289823)

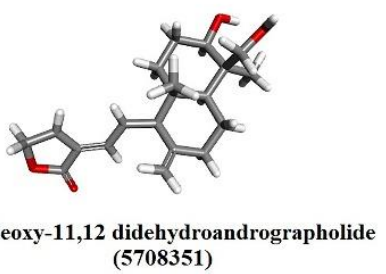

Andrographidine C (5318484)

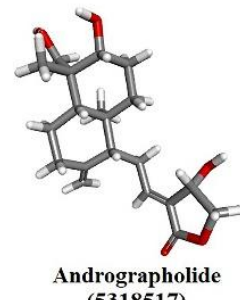
(5318517)

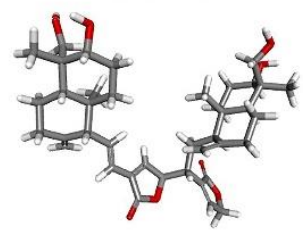

Bisandrographolide (12000062)
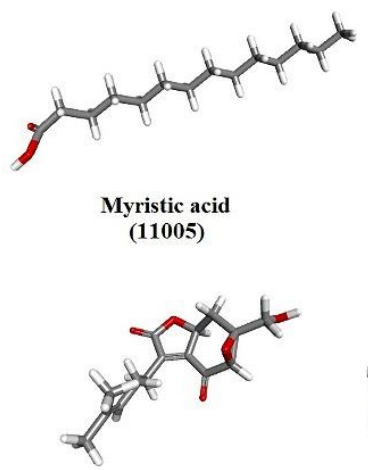

Paniculide C
$(101289824)$

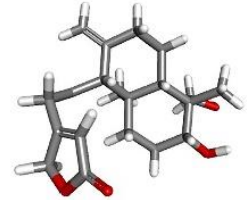

14-deoxy-11-oxoandrographolide (101593061)

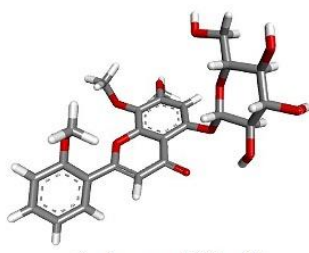

Andrographidine E (13963769)
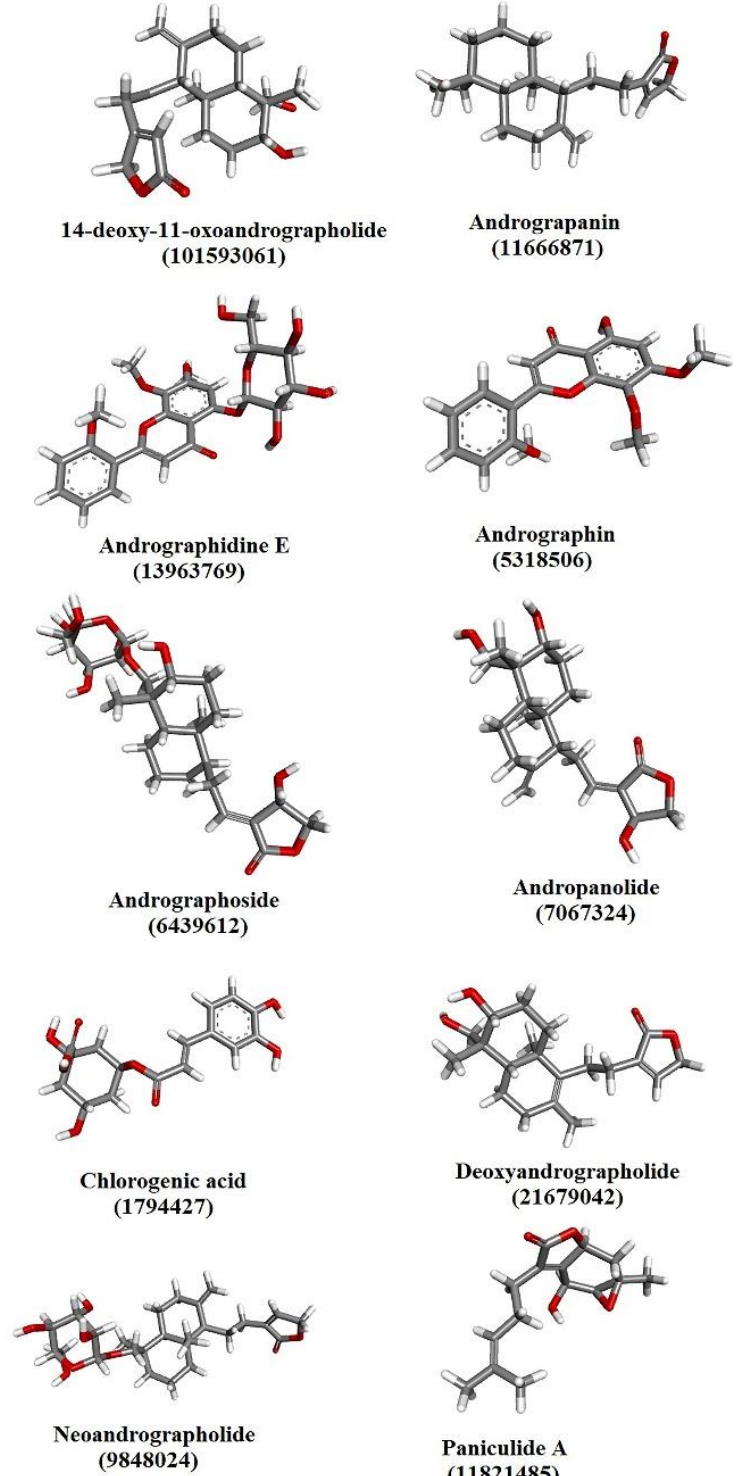

Andrographin (5318506)
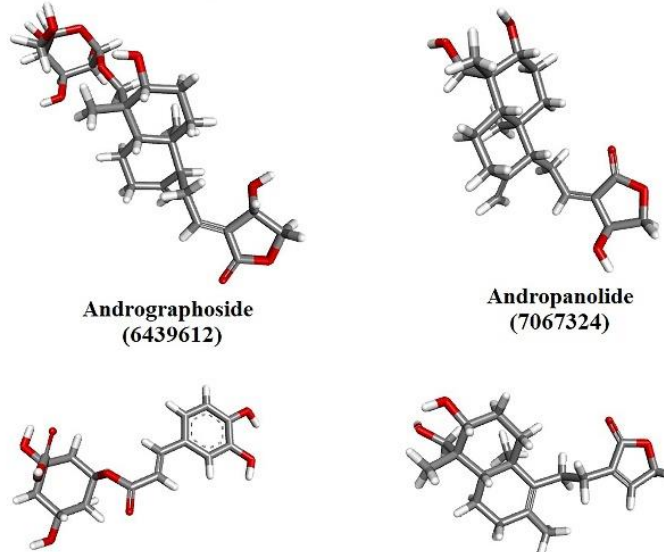

Chlorogenic acid (1794427)

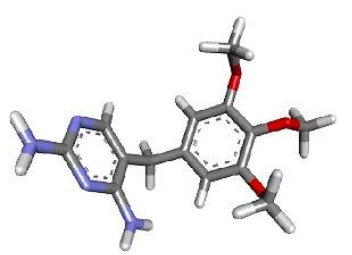

$\underset{(5578)}{\text { Trimethoprim }}$

Figure 1. Three-dimensional structure of ligands.

TMP was clinically introduced in 1968, and the S.aureus TMP resistance was first reported in 1980s [9]. Trimethoprim resistance in $S$. aureus may raise from the chromosomal gene (dfrB) mutations or introduction of dfrA, dfrG, dfrK naturally occurring resistant genes via plasmid $[10,11]$. Since the 1940 s, the topical application of sulfa powder to soldier's wounds at the battlefield for inhibition of the folate biosynthetic pathway to prevent infection has been successful. Folate pathway inhibition using 'Antifolate' results in thymine-less cell death of the bacteria [12]. Dihydrofolate reductase (DHFR) is a folate-dependent enzyme 
involved in various cellular components biosynthesis [13]. Hence, DHFR is considered a potential target to address the AMR towards S.aureus infection. Andrographis paniculata, an annual herb of the Acanthaceae family, is traditionally used for treating various ailments, including microbial infections. The plant has been reported for its antibacterial, antiviral, antiinflammatory, antioxidant, and antipyretic activity [14]. Previously we have reported the presence of phytochemicals that could potentially inhibit PLAA2, a major toxic component of snake venom (15). In the present study, we propose to identify novel plant-based antifolates through computational analysis.

\section{Materials and Methods}

\subsection{Preparation of ligands and protein.}

Structures of twenty-two phytochemicals of A. paniculata and reference compound trimethoprim - a known inhibitor of DHFR were obtained from PubChem in SDF format (https://pubchem.ncbi.nlm.nih.gov/) and converted to PDB format using Marvin view tool. Three-dimensional structures of ligands, along with their PubChem ID are presented as Fig.1.

The PDB structure of target protein S.aureus Dihydrofolate reductase wild type (SaDHFR) (PDB ID: 3FRE) and mutated (SaDHFR F98Y) (PDB ID: 3FRB) were retrieved from Protein Data Bank (https://www.rcsb.org/). The three-dimensional structure of target protein S.aureus Dihydrofolate reductase complexed with NADPH and TMP are presented as Fig. 2.

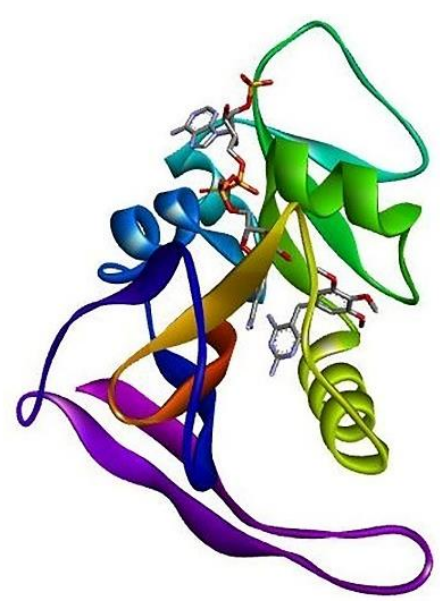

Figure 2. Three-dimensional structure of Dihydrofolate reductase complexed with NADPH and TMP.

\subsection{Molecular docking analysis using Autodock.}

The binding efficiency of $A$. paniculata phytochemicals with the DHFR active site was predicted using Autodock 4, an In Silico method. Autodock 4 uses the combination of mathematical calculations and algorithms to find the binding probability of a ligand to a protein or peptide. Autodock 4 advantage is side-chain flexibility in the protein during the ligand docking process. Autodock 4 has a free energy scoring function that works based on linear regression analysis, AMBER force field, and an even large set of varied complexes of proteinligand with well-known inhibition constants that were used in the previous version of autodock - Autodock 3.0.

Water molecules were removed, followed by polar hydrogen bonds and Kollman charges to the target protein. Then the number of torsions was set to the ligand. Both the target protein and ligand were saved in pdbqt file format. For a ligand to bind at the target protein's 
active site, a grid map was assigned with $\mathrm{x}, \mathrm{y}, \mathrm{z}$ points as $24.778,11.673$, and 38.803, respectively. Docking was performed using the Lamarckian genetic algorithm. Binding energy, binding residues, inhibition constant analyzed and produced as docking output [16].

\subsection{Visualization using BIOVIA Discovery Studio Visualizer.}

Docked complex of A.paniculata phytochemicals with target proteins SaDHFR and SaDHFR F98Y were visualized and analyzed using BIOVIA Discovery Studio Visualizer. It is a visualization and analysis suite for public use. The visualizer has many features like macromolecule design, ligand, and structure-based design and visualization. BIOVIA Discovery Studio Visualizer is an interactive three-dimensional simulation tool for visualizing and analyzing the crystal structure of small molecules, proteins, and nucleic acid.

\section{Results and Discussion}

Trimethoprim is a commonly used antibiotic categorized under the "Access" group of AWaRe (Access, Watch and Reserve) of antibiotics by WHO. Which is one among the top 5 antibiotics consumed worldwide [17], and also in combination with sulfamethoxazole, TMP is the 2nd top-selling FDC (Fixed-Dose Combination) in India [18]. The foremost reason behind the high consumption of TMP is its efficiency and then its least side effect. A quest on the biosimilar for trimethoprim was performed on the naturally occurring plant secondary metabolites was analyzed for potential targeting DHFR.

DHFR is a vital enzyme for all living organisms. DHFR catalyzes dihydrofolate (DHF) conversion to tetrahydrofolate (THF) using NADPH as a cofactor. Hence antifolates are one of the potential interests for addressing various diseases, especially cancer other than microbial infections. Antifolates comprise a large family of diversified compounds. Antifolates are used in treating a wide range of diseases, namely methotrexate (MTX), trimetrexate (TMTX) for cancer, trimethoprim (TMP), WR99210 for bacterial and Pyrimethamine (PYR), cycloguanil (CYC) for protozoal infection [19-23]. The folate biosynthetic pathway delivers a key strategy for designing and developing antifolates for controlling the growth of bacteria [24]. Antifolates act as a competitive inhibitor by binding at the folate-binding site of DHFR. TMP binds 2500 folds more tightly to bacterial DHFR than human DHFR [25]. Moreover, human purine synthesis depends on dietary folic acid, not endogenous folic acid; hence the host purine synthesis is not affected by trimethoprim. Antimicrobial resistance (AMR) towards trimethoprim by the $S$. aureus is due to a single amino acid substitution at the 98th position PHE to TYR, in DHFR. The mutation increased the resistance 64-fold in trimethoprim MIC $[26,27]$. Hence the DHFR is identified as a potential target for antimicrobial drug development. Indigenous traditional medicinal plants are gaining importance for their remarkable efficacy in treating various diseases. Many medicinal plant extracts have been reported for their antimicrobial activity [28-32]. In the present work, the binding efficiency and interaction of various phytochemicals of $A$. paniculata with the wild type dihydrofolate reductase of SaDHFR and mutated SaDHFR F98Y of S.aureus was studied by molecular docking and in silico method using AUTODOCK 4 tool, which is largely used and has worldwide acceptance.

A.paniculata phytochemicals binding energy, inhibition constant, and interacting amino acids with SaDHFR and SaDHFR F98Y are tabulated in Table 1

Active site residues of $S$. aureus DHFR comprise LEU5, VAL6, LEU20, LEU28, VAL31, THR46, ILE-50, and LEU54 [33]. In this study, the analysis revealed the non-covalent 
interactions of $A$. paniculata phytochemicals with active site amino acids of SaDHFR and SaDHFR F98Y. Bisandrographolide is the only compound that failed to show binding affinity though it interacted with active site amino acids. Two-dimensional representation of ligands (A.paniculata phytochemicals and trimethoprim) interaction with different targets, namely SaDHFR and SaDHFR F98Y is shown in Fig 3 and 4, respectively.

Table 1. A. paniculata phytochemicals and reference compound trimethoprim binding energy, inhibition constant, and interacting amino acids with SaDHFR and SaDHFR F98Y.

\begin{tabular}{|c|c|c|c|c|c|c|c|}
\hline \multirow[t]{2}{*}{ S.No } & \multirow[t]{2}{*}{ Ligand name } & \multicolumn{3}{|c|}{ SaDHFR (PDB ID:3FRE) } & \multicolumn{3}{|c|}{ SaDHFR F98Y (PDB ID: 3FRB) } \\
\hline & & $\begin{array}{l}\text { Binding } \\
\text { Energy } \\
\text { (kCal/mol) }\end{array}$ & $\begin{array}{l}\text { Inhibition } \\
\text { constant }\end{array}$ & $\begin{array}{l}\text { Interacting } \\
\text { amino acids }\end{array}$ & $\begin{array}{l}\text { Binding } \\
\text { Energy } \\
\text { (kCal/mol) }\end{array}$ & $\begin{array}{l}\text { Inhibition } \\
\text { constant }\end{array}$ & $\begin{array}{l}\text { Interacting } \\
\text { amino acids }\end{array}$ \\
\hline 1 & $\begin{array}{l}\text { 14- } \\
\text { acetylandrographol } \\
\text { ide }\end{array}$ & -9.77 & $68.92 \mathrm{nM}$ & $\begin{array}{l}\text { THR121 } \\
\text { LEU5 } \\
\text { ASN18 }\end{array}$ & -9.79 & $66.34 \mathrm{nM}$ & $\begin{array}{l}\text { ALA7 ASN18 } \\
\text { GLN95 } \\
\text { TYR98 } \\
\text { LEU5 } \\
\text { LEU20 } \\
\text { LYS45 }\end{array}$ \\
\hline 2 & $\begin{array}{l}\text { 14-deoxy- } \\
\text { 11,12didehydroand } \\
\text { rographolide }\end{array}$ & -9.55 & $100.64 \mathrm{nM}$ & $\begin{array}{l}\text { ALA7 } \\
\text { ASN18 } \\
\text { THR121 } \\
\text { LEU20 } \\
\text { LYS45 } \\
\end{array}$ & -9.59 & $92.81 \mathrm{nM}$ & $\begin{array}{l}\text { ALA7 ASN18 } \\
\text { LEU20 } \\
\text { LYS45 } \\
\text { TYR98 }\end{array}$ \\
\hline 3 & $\begin{array}{l}\text { 14-deoxy-11- } \\
\text { oxoandrographolid } \\
\text { e }\end{array}$ & -10.31 & $27.82 \mathrm{nM}$ & $\begin{array}{l}\text { LEU5 } \\
\text { ALA7 } \\
\text { PHE92 } \\
\text { GLN95 } \\
\text { THR121 } \\
\text { ILE14 } \\
\text { LEU20 } \\
\text { ILE50 } \\
\end{array}$ & -10.11 & $39.0 \mathrm{nM}$ & $\begin{array}{l}\text { ALA7 } \\
\text { SER49 } \\
\text { PHE92 } \\
\text { GLN95 } \\
\text { THR121 } \\
\text { LEU20 } \\
\text { ILE50 }\end{array}$ \\
\hline 4 & Andrograpanin & -9.76 & $70.25 \mathrm{nM}$ & $\begin{array}{l}\text { ALA7 } \\
\text { ASN18 } \\
\text { ASP120 } \\
\text { VAL6 } \\
\text { ILE14 } \\
\text { LEU20 } \\
\end{array}$ & -9.81 & $64.84 \mathrm{nM}$ & $\begin{array}{l}\text { ASN18 } \\
\text { ILE14 } \\
\text { LEU20 } \\
\text { LYS45 } \\
\text { PHE92 }\end{array}$ \\
\hline 5 & Andrographdine A & -1.69 & $57.66 \mathrm{mM}$ & $\begin{array}{l}\text { LEU5 } \\
\text { ALA7 } \\
\text { PHE92 } \\
\text { ASP27 } \\
\text { ILE50 } \\
\text { LEU20 }\end{array}$ & -1.54 & $74.53 \mathrm{mM}$ & $\begin{array}{l}\text { LEU5 } \\
\text { ALA7 } \\
\text { ASP27 } \\
\text { PHE92 } \\
\text { LEU20 } \\
\text { ILE50 } \\
\text { GLN19 }\end{array}$ \\
\hline 6 & Andrographidine $\mathrm{C}$ & -6.89 & $8.85 \mathrm{uM}$ & $\begin{array}{l}\text { ALA7 } \\
\text { ASP27 } \\
\text { PHE92 } \\
\text { LEU28 } \\
\text { LEU20 } \\
\text { ILE50 }\end{array}$ & -6.06 & $35.98 \mu \mathrm{M}$ & $\begin{array}{l}\text { ALA7 } \\
\text { ASP27 } \\
\text { TYR98 } \\
\text { PHE92 } \\
\text { ILE50 } \\
\text { ILE50 } \\
\text { LEU20 } \\
\text { LEU28 }\end{array}$ \\
\hline 7 & Andrographidine $\mathrm{E}$ & -6.22 & $27.5 \mathrm{uM}$ & $\begin{array}{l}\text { ALA7 } \\
\text { ASP27 } \\
\text { PHE92 } \\
\text { ILE50 } \\
\text { LEU20 } \\
\text { LEU28 } \\
\text { SER49 }\end{array}$ & -6.07 & $35.77 \mu \mathrm{M}$ & $\begin{array}{l}\text { ALA7 } \\
\text { ASP27 } \\
\text { TYR98 } \\
\text { GLN19 } \\
\text { PHE92 } \\
\text { IlE50 } \\
\text { LEU20 } \\
\text { LEU28 }\end{array}$ \\
\hline 8 & Andrographin & -7.2 & $5.29 \mathrm{uM}$ & $\begin{array}{l}\text { ALA7 } \\
\text { ILE14 } \\
\text { LEU20 } \\
\text { PHE92 }\end{array}$ & -8.0 & $1.36 \mu \mathrm{M}$ & $\begin{array}{l}\text { SER49 } \\
\text { ALA7 } \\
\text { ILE14 } \\
\text { LEU20 } \\
\text { PHE92 }\end{array}$ \\
\hline 9 & Andrographiside & -4.87 & $267.95 \mathrm{uM}$ & $\begin{array}{l}\text { ASN18 } \\
\text { SER49 }\end{array}$ & -1.94 & $37.81 \mathrm{mM}$ & $\begin{array}{l}\text { ASN18 } \\
\text { ASP27 }\end{array}$ \\
\hline
\end{tabular}




\begin{tabular}{|c|c|c|c|c|c|c|c|}
\hline \multirow[t]{2}{*}{ S.No } & \multirow[t]{2}{*}{ Ligand name } & \multicolumn{3}{|c|}{ SaDHFR (PDB ID:3FRE) } & \multicolumn{3}{|c|}{ SaDHFR F98Y (PDB ID: 3FRB) } \\
\hline & & $\begin{array}{l}\text { Binding } \\
\text { Energy } \\
\text { (kCal/mol) } \\
\end{array}$ & $\begin{array}{l}\text { Inhibition } \\
\text { constant }\end{array}$ & $\begin{array}{l}\text { Interacting } \\
\text { amino acids }\end{array}$ & $\begin{array}{l}\text { Binding } \\
\text { Energy } \\
(\mathrm{kCal} / \mathrm{mol}) \\
\end{array}$ & $\begin{array}{l}\text { Inhibition } \\
\text { constant }\end{array}$ & $\begin{array}{l}\text { Interacting } \\
\text { amino acids }\end{array}$ \\
\hline & & & & $\begin{array}{l}\text { GLN95 } \\
\text { ASP120 } \\
\text { THR121 } \\
\text { VAL6 } \\
\text { ILE14 } \\
\text { LEU20 } \\
\text { PHE98 }\end{array}$ & & & $\begin{array}{l}\text { GLN95 ILE14 } \\
\text { LEU20 } \\
\text { ILE50 } \\
\text { PHE92 } \\
\text { TYR98 }\end{array}$ \\
\hline 10 & Andrographolide & -9.31 & $150.02 \mathrm{nM}$ & $\begin{array}{l}\text { PHE202 } \\
\text { THR121 } \\
\text { GLY15 } \\
\text { ILE14 } \\
\text { LEU20 } \\
\text { LYS45 }\end{array}$ & -9.18 & $186.97 \mathrm{nM}$ & $\begin{array}{l}\text { ALA7 ASN18 } \\
\text { PHE92 } \\
\text { VAL6 } \\
\text { ILE14 } \\
\text { LEU20 } \\
\text { LYS45 } \\
\text { TYR98 }\end{array}$ \\
\hline 11 & Andrographoside & -5.69 & $67.2 \mathrm{uM}$ & $\begin{array}{l}\text { ASN18 } \\
\text { GLN95 } \\
\text { THR46 } \\
\text { THR121 } \\
\text { VAL6 } \\
\text { ILE14 } \\
\text { LEU20 } \\
\text { PHE98 } \\
\end{array}$ & -3.31 & $3.76 \mathrm{nM}$ & $\begin{array}{l}\text { ASP27 } \\
\text { ASP120 } \\
\text { ILE14 } \\
\text { LEU20 } \\
\text { ILE50 }\end{array}$ \\
\hline 12 & Andropanolide & -8.34 & $764.41 \mathrm{nM}$ & $\begin{array}{l}\text { ASP27 } \\
\text { THR46 } \\
\text { SER49 } \\
\text { VAL6 } \\
\text { ALA7 } \\
\text { LEU20 } \\
\text { LEU28 } \\
\text { VAL31 } \\
\text { PHE92 } \\
\text { PHE98 } \\
\end{array}$ & -9.34 & $142.93 \mathrm{nM}$ & $\begin{array}{l}\text { ALA7 ASN18 } \\
\text { PHE92 } \\
\text { LEU20 } \\
\text { LYS45 } \\
\text { TYR98 }\end{array}$ \\
\hline 13 & Andropanoside & -8.34 & $764.41 \mathrm{nM}$ & $\begin{array}{l}\text { ASP27 } \\
\text { THR46 } \\
\text { SER49 } \\
\text { VAL6 } \\
\text { ALA7 } \\
\text { LEU20 } \\
\text { LEU28 } \\
\text { VAL31 } \\
\text { PHE92 } \\
\text { PHE98 }\end{array}$ & -6.22 & $27.62 \mu \mathrm{M}$ & $\begin{array}{l}\text { ASN18 } \\
\text { SER49 } \\
\text { LEU5 } \\
\text { VAL6 } \\
\text { ILE14 VAL31 } \\
\text { PHE92 } \\
\text { TYR98 }\end{array}$ \\
\hline 14 & Bisandrographolide & 35.06 & - & $\begin{array}{l}\text { ASN18 } \\
\text { TRP22 } \\
\text { LEU5 } \\
\text { LEU24 } \\
\text { LEU28 } \\
\text { PRO25 } \\
\text { ILE50 } \\
\text { PHE98 } \\
\text { HIS23 } \\
\text { VAL6 } \\
\text { LEU20 } \\
\text { VAL31 }\end{array}$ & 84.96 & - & $\begin{array}{l}\text { ALA7 } \\
\text { ILE14 } \\
\text { LEU20 } \\
\text { LEU28 } \\
\text { THR46 } \\
\text { LEU54 } \\
\text { PHE92 } \\
\text { GLY94 } \\
\text { LEU5 } \\
\text { VAL31 } \\
\text { ILE50 } \\
\text { TYR98 } \\
\text { PHE92 }\end{array}$ \\
\hline 15 & Chlorogenic acid & -5.89 & $48.52 \mathrm{uM}$ & $\begin{array}{l}\text { LEU5 } \\
\text { ASN18 } \\
\text { ASP27 } \\
\text { PHE92 }\end{array}$ & -5.34 & $121.34 \mu \mathrm{M}$ & $\begin{array}{l}\text { LEU5 } \\
\text { ALA7 } \\
\text { ASN18 } \\
\text { ASP27 } \\
\text { PHE92 } \\
\text { TYR98 } \\
\end{array}$ \\
\hline 16 & $\begin{array}{l}\text { Deoxyandrographo } \\
\text { lide }\end{array}$ & -9.28 & $158.5 \mathrm{nM}$ & $\begin{array}{l}\text { ALA7 } \\
\text { ASN18 } \\
\text { ILE14 } \\
\text { LEU20 }\end{array}$ & -9.31 & $148.89 \mathrm{nM}$ & $\begin{array}{l}\text { ALA7 ASN18 } \\
\text { ILE14 } \\
\text { LEU20 } \\
\text { LYS45 } \\
\text { ILE50 }\end{array}$ \\
\hline
\end{tabular}




\begin{tabular}{|c|c|c|c|c|c|c|c|}
\hline \multirow[t]{2}{*}{ S.No } & \multirow[t]{2}{*}{ Ligand name } & \multicolumn{3}{|c|}{ SaDHFR (PDB ID:3FRE) } & \multicolumn{3}{|c|}{$\begin{array}{l}\text { SaDHFR F98Y (PDB ID: 3FRB) } \\
\end{array}$} \\
\hline & & $\begin{array}{l}\text { Binding } \\
\text { Energy } \\
(\mathrm{kCal} / \mathrm{mol})\end{array}$ & $\begin{array}{l}\text { Inhibition } \\
\text { constant }\end{array}$ & $\begin{array}{l}\text { Interacting } \\
\text { amino acids }\end{array}$ & $\begin{array}{l}\text { Binding } \\
\text { Energy } \\
(\mathrm{kCal} / \mathrm{mol})\end{array}$ & $\begin{array}{l}\text { Inhibition } \\
\text { constant }\end{array}$ & $\begin{array}{l}\text { Interacting } \\
\text { amino acids }\end{array}$ \\
\hline & & & & $\begin{array}{l}\text { VAL31 } \\
\text { LYS45 } \\
\text { ILE50 } \\
\text { PHE92 } \\
\end{array}$ & & & $\begin{array}{l}\text { ALA7 } \\
\text { TYR98 }\end{array}$ \\
\hline 17 & Isoandrographolide & -9.37 & $135.36 \mathrm{nM}$ & $\begin{array}{l}\text { ALA7 } \\
\text { GLN19 } \\
\text { THR121 } \\
\text { LEU5 } \\
\text { ILE14 } \\
\text { LEU20 } \\
\text { VAL31 } \\
\text { PHE92 } \\
\end{array}$ & -9.38 & $132.55 \mathrm{nM}$ & $\begin{array}{l}\text { ALA7 } \\
\text { PHE92 } \\
\text { GLN95 } \\
\text { ASN18 } \\
\text { ILE14 } \\
\text { LEU20 } \\
\text { LYS45 } \\
\text { TYR98 } \\
\end{array}$ \\
\hline 18 & Myristic acid & -4.86 & 274.31uM & $\begin{array}{l}\text { LEU28 } \\
\text { ARG57 }\end{array}$ & -4.71 & $354.06 \mu \mathrm{M}$ & $\begin{array}{l}\text { ASN18 } \\
\text { LYS45 } \\
\text { LEU5 } \\
\text { ALA7 } \\
\text { VAL31 }\end{array}$ \\
\hline 19 & $\begin{array}{l}\text { Neoandrographolid } \\
\text { e }\end{array}$ & -7.78 & $1.97 \mathrm{uM}$ & $\begin{array}{l}\text { ASN18 } \\
\text { LYS45 } \\
\text { GLN95 } \\
\text { VAL6 } \\
\text { ILE14 } \\
\text { LEU20 } \\
\text { LEU28 } \\
\text { VAL31 } \\
\text { PHE92 } \\
\text { PHE98 }\end{array}$ & -6.93 & $8.29 \mu \mathrm{M}$ & $\begin{array}{l}\text { VAL6 } \\
\text { ILE14 } \\
\text { LEU20 } \\
\text { LEU28 } \\
\text { VAL31 } \\
\text { PHE92 } \\
\text { TYR98 }\end{array}$ \\
\hline 20 & Paniculide A & -7.51 & $3.1 \mathrm{uM}$ & $\begin{array}{l}\text { ALA7 } \\
\text { PHE92 } \\
\text { THR46 } \\
\text { LEU5 } \\
\text { ILE14 } \\
\text { LEU20 } \\
\text { VAL31 }\end{array}$ & -7.39 & $3.84 \mu \mathrm{M}$ & $\begin{array}{l}\text { ALA7 } \\
\text { PHE92 } \\
\text { LEU20 } \\
\text { VAL31 }\end{array}$ \\
\hline 21 & Paniculide B & -7.35 & $4.08 \mathrm{uM}$ & $\begin{array}{l}\text { ALA7 } \\
\text { PHE92 } \\
\text { LEU5 } \\
\text { ILE14 } \\
\text { LEU20 } \\
\text { VAL31 }\end{array}$ & -7.11 & $6.18 \mu \mathrm{M}$ & $\begin{array}{l}\text { ALA7 } \\
\text { PHE92 } \\
\text { ILE14 } \\
\text { LEU5 } \\
\text { LEU20 } \\
\text { VAL31 }\end{array}$ \\
\hline 22 & Paniculide C & -7.2 & $5.29 \mathrm{uM}$ & $\begin{array}{c}\text { ALA7 } \\
\text { ILE14 } \\
\text { LEU20 } \\
\text { PHE92 }\end{array}$ & -7.25 & $4.84 \mu \mathrm{M}$ & $\begin{array}{l}\text { ALA7 } \\
\text { SER49 } \\
\text { LEU5 } \\
\text { ILE14 } \\
\text { LEU20 } \\
\text { VAL31 } \\
\text { PHE92 } \\
\end{array}$ \\
\hline 23 & Trimethoprim & -7.64 & $2.53 \mathrm{uM}$ & $\begin{array}{l}\text { ALA7 } \\
\text { ILE14 } \\
\text { ASP27 } \\
\text { THR121 } \\
\text { PHE92 } \\
\text { VAL6 } \\
\text { LEU20 }\end{array}$ & -7.96 & $1.48 \mu \mathrm{M}$ & $\begin{array}{l}\text { ALA7 } \\
\text { ILE14 } \\
\text { ASP27 } \\
\text { VAL6 } \\
\text { LEU20 } \\
\text { PHE92 } \\
\text { GLY15 }\end{array}$ \\
\hline
\end{tabular}

TYR98 of SaDHFR F98Y formed a conventional hydrogen bond with 14acetylandrographolide, Andrographidine $\mathrm{C}$ and E, van der Waals interaction with 14-deoxy11,12didehydroandrographolide, Andrographolide, Andropanolide, Chlorogenic acid, Deoxyandrographolide and pi-alkyl interaction with Andrographiside, Andropanoside, Bisandrographolide and Neoandrographolide, whereas PHE98 of SaDHFR formed an only pialkyl bond with Andrographiside, Andrographoside, Andropanolide, Andropanoside, bisandrographolide, and Neoandrographolide. 


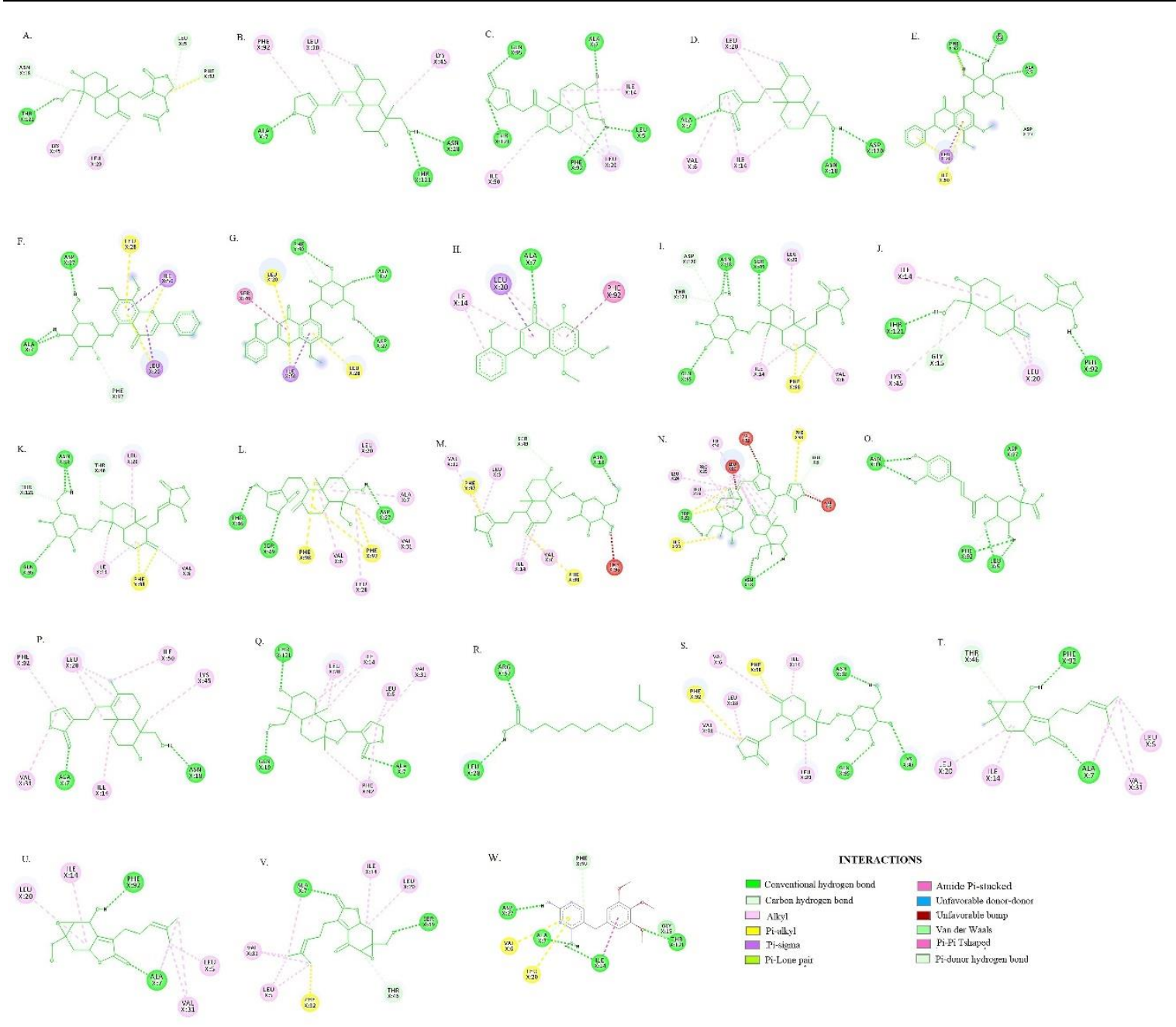

Figure 3. Two-dimensional representation of Staphylococcus aureus wild type Dihydrofolate reductase (SaDHFR) residues interaction with ligands. A.14-acetylandrographolide, B. 14-deoxy11,12didehydroandrographolide, C. 14-deoxy-11-oxoamdrographolide, D. Andrograpanin, E. Andrographidine A, F. Andrographidine C, G. Andrographidine E, H. Andrographin, I. Andrographiside, J. Andrographolide, K. Andrographoside, L. Andropanolide, M. Andropanoside, N. Bisandrographolide, O. Chlorogenic acid, P. Deoxyandrographolide, Q. Isoandrographolide, R. Myristic acid, S. Neoandrographolide, T. Paniculide A, U. Paniculide B, V. Paniculide C, W. Trimethoprim.

DHFR structural analysis studies by Bhosle et al. 2016, GLN95 residue at supersite a druggable space in a substructure of $2 \mathrm{~W} 9 \mathrm{H}$ (Wild-type $S$. aureus DHFR in complex with trimethoprim) were unique when compared with all other clusters of supersites [34]. In our present study, phytochemicals formed a conventional hydrogen bond with the target.

14-deoxy-11-oxoandrographolide and Andrographiside formed a conventional hydrogen bond with GLN95 of both SaDHFR wild type and SaDHFR F98Y mutated whereas 14-acetylandrographolide, Isoandrographolide interacting only with SaDHFR F98Y through a conventional hydrogen bond. Andrographiside and Neoandrographolide interacted with SaDHFR.

ALA at $7^{\text {th }}$ position of DHFR is shown to be a target for several phytochemicals isolated from plants and biomolecules like Chlorogenic acid, Ellagic acid, Gallic acid, Hippuric acid and Clavulanic acid [35]. Similarly, in our study, phytochemicals bind with ALA7 of SaDHFR and SaDHFR F98Y. 


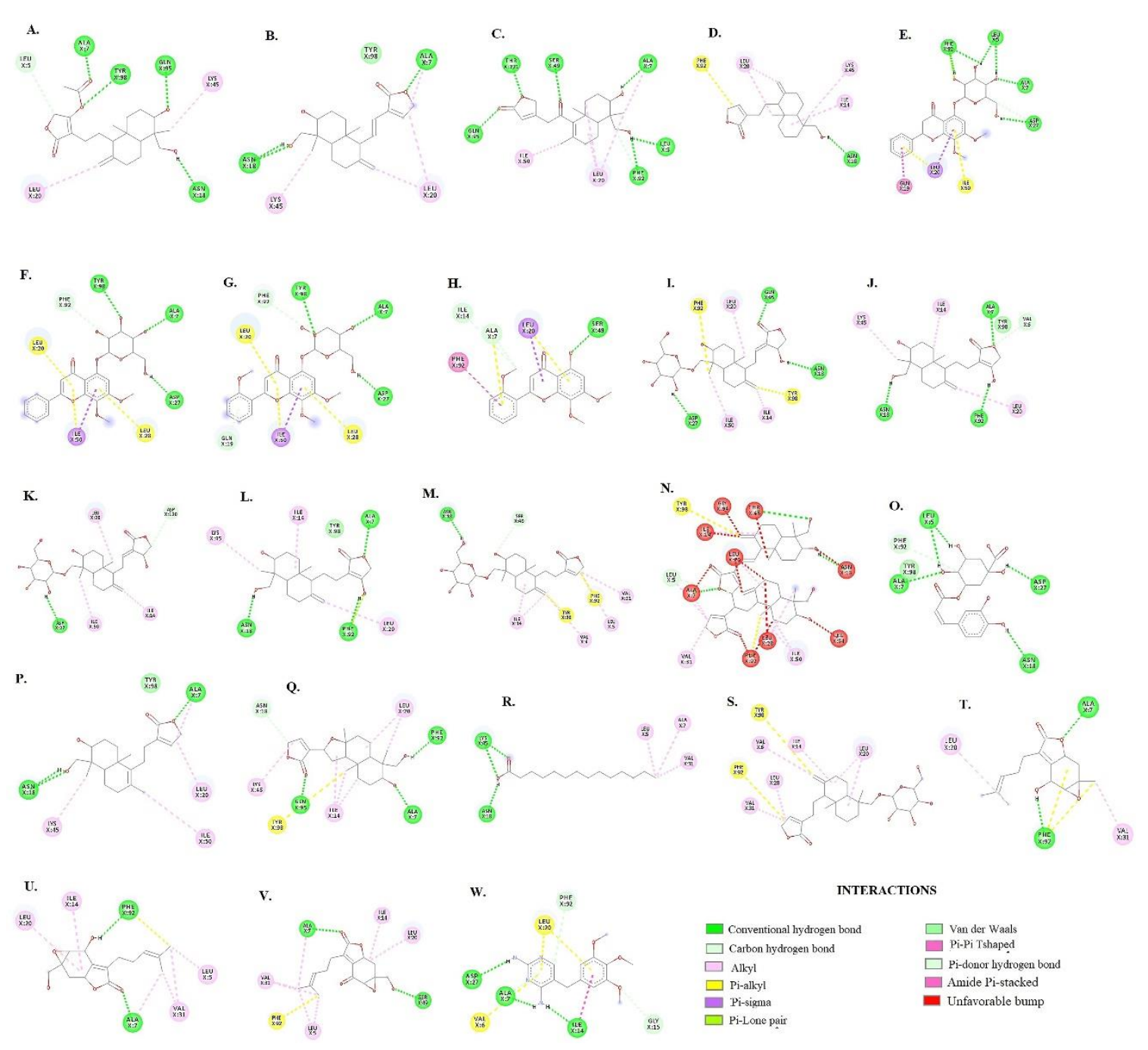

Figure 4. Two-dimensional representation of Staphylococcus aureus mutated Dihydrofolate reductase (SaDHFR F98Y) residues interaction with ligands. A.14-acetylandrographolide, B. 14-deoxy11,12didehydroandrographolide, C. 14-deoxy-11-oxoamdrographolide, D. Andrograpanin, E. Andrographidine A, F. Andrographidine C, G. Andrographidine E, H. Andrographin, I. Andrographiside, J. Andrographolide, K. Andrographoside, L. Andropanolide, M. Andropanoside, N. Bisandrographolide, O. Chlorogenic acid, P. Deoxyandrographolide, Q. Isoandrographolide, R. Myristic acid, S. Neoandrographolide, T. Paniculide A, U. Paniculide B, V. Paniculide C, W. Trimethoprim.

The medicinal property of $A$. paniculata is well known by the scientific community and herbal medicine practitioners', of which the antimicrobial property of A. paniculata was reported by several scientific groups against various pathogens [36-38]. Ali et al. reported the inhibitory activity of A.paniculata methanolic extract against S.aureus and E.coli growth [39]. Mishra et al. 2009 reported the antibacterial activity of $A$. paniculata ethanol extract against both Gram-negative and Gram-positive bacteria [40]. Leaf extracts of A. paniculata showed high antimicrobial activity at $200 \mathrm{mg} / \mathrm{ml}$ concentration against gram-positive bacteria $B$. cereus and S.aureus [41]. Antimicrobial activity of A. paniculata crude methanol extract and its fractions were evaluated against clinical pathogens E. cloacae, E.coli, S.typhi, S.aureus, and C. albicans. All the extracts exhibited antimicrobial activity against all pathogens except E.coli [42]. In a similar study, aqueous and different solvent leaf extracts of $A$. paniculata showed inhibitory activity against UTI (Urinary Tract Infection) bacteria [43]. Root, stem and leaves of A. paniculata in different solvent extracts studied for antibacterial activity against human pathogens - Staphylococcus sp.; E.coli, Salmonella typhi, Pseudomonas sp. Methanol extract exhibited high activity against E.coli followed by S.typhi, Pseudomonas sp.; and 
Staphylococcus sp. [44]. Whole plant A. paniculata dichloromethane (non-polar solvent), methyl alcohol, and water (polar solvent) extracts were tested for antimicrobial activity by disc diffusion method at different concentrations $(250,500,1000 \mu \mathrm{g} / \mathrm{ml})$ against skin diseasecausing seven Gram-positive and five Gram-negative bacteria. Extracts showed substantial antimicrobial activity against both Gram-positive and Gram-negative bacteria [45]. Antibacterial activity of polar and non-polar leaf extracts of A.paniculata evaluated against the S.aureus, S.pyrogenes, E.coli, and S.typhi. Methanol extracts showed greater inhibitory activity than hexane extract [46]. A.paniculata aqueous extract, Andrographolide, the main constituent of the A.paniculata, and arabinogalactan protein from A.paniculata (dried form) exhibited antibacterial activity against B.subtilis, P.aeruginosa and E.coli [47]. Methanol and chloroform extracts of A.paniculata showed antibacterial activity against clinical pathogens [48].

Hence there exists strong evidence for the antimicrobial property of A. paniculata. The efficiency of phytochemicals of $A$. paniculata binding both wild type and the common mutated strain shows the possibility of solving AMR issues and problems.

The molecular mechanism of $A$. paniculata antimicrobial property and the identification of phytochemicals mediating the potent bactericidal property has not been previously reported. Hence results of the present study are the first report giving a clue on the drug target, i.e., DHFR, and the potential phytochemicals. The current result may also provoke the scientific groups to conduct further biochemical and pharmacological studies on the compounds and the whole extract of A. paniculata targeting DHFR, therefore addressing AMR's global issue.

\section{Conclusions}

AMR is a rising global issue that needs serious consideration. The probability and possibility of solving AMR with advanced technology are meager and also may require a huge budget, whereas the whole plant extracts containing numerous phytochemicals acting synergistically or non-synergistically on more than one target of infectious pathogens may address AMR shortly. Phytochemicals of A. paniculata have the potential to address AMR by targeting DHFR.

\section{Funding}

This research received no external funding.

\section{Acknowledgments}

Authors thank School of Life Sciences, B.S.Abdur Rahman Crescent Institute of Science and Technology for providing fellowship and research facility.

\section{Conflicts of Interest}

The authors declare no conflict of interest.

\section{References}

1. Tong, S.Y.; Davis, J.S.; Eichenberger, E.; Holland, T.L.; Fowler, V.G Jr. Staphylococcus aureus infections: epidemiology, pathophysiology, clinical manifestations and management. Clin Microbiol Rev 2015, 2, https://doi.org/10.1128/CMR.00134-14. 
2. Theos, K.R.; Johnson, K.M.; Johnson, D.W. Staphylococcus aureus Antibiotic Susceptibilities in Infections in an Outpatient Dermatology Office on O'ahu. Hawai'i journal of medicine \& public health : a journal of Asia Pacific Medicine \& Public Health 2019, 78, 163-168.

3. Horino, T.; Hori, S. Metastatic infection during Staphylococcus aureus bacteremia. Journal of Infection and Chemotherapy 2020, 26, 162-169, https://doi.org/10.1016/j.jiac.2019.10.003.

4. Hagihara, M.; Kato, H.; Uchida, S.; Yamashita, R.; Tanaka, S.; Sakanashi, D.; Shiota, A.; Asai, N.; Suematsu, Y.; Namiki, N.; Mikano, H. The First Report on Pharmacokinetic/Pharmacodynamic Study of Trimethoprim/Sulfamethoxazole against Staphylococcus aureus with a Neutropenic Murine Thigh Infection Model. Chemotherapy 2019, 64, 224-232, https://doi.org/10.1159/000507540.

5. Kluytmans, J.; van Belkum, A.; Verbrugh, H. Nasal carriage of Staphylococcus aureus: epidemiology, underlying mechanisms, and associated risks. Clinical Microbiology Reviews 1997, 10, 505-20, http://doi.org/10.1128/CMR.10.3.505.

6. Chambers, H.F.; DeLeo, F.R. Waves of resistance: Staphylococcus aureus in the antibiotic era. Nature Reviews Microbiology 2009, 7, 629-641, http://doi.org/10.1038/nrmicro2200.

7. Yu, Z.; Tang, J.; Khare, T.; Kumar, V. The alarming antimicrobial resistance in ESKAPEE pathogens: Can essential oils come to the rescue? Fitoterapia 2020, 140, https://doi.org/10.1016/j.fitote.2019.104433.

8. He, J.; Qiao, W.; An, Q.; Yang, T.; Luo, Y. Dihydrofolate reductase inhibitors for use as antimicrobial agents. European Journal of Medicinal Chemistry 2020, 195, https://doi.org/10.1016/j.ejmech.2020.112268.

9. Reeve, S.M.; Si, D.; Krucinska, J.; Yan, Y.; Viswanathan, K.; Wang, S.; Holt, G.T.; Frenkel, M.S.; Ojewole, A.A.; Estrada, A.; Agabiti, S.S.; Alverson, J.B.; Gibson, N.D.; Priestley, N.D.; Wiemer, A.J.; Donald, B.R.; Wright, D.L. Toward Broad Spectrum Dihydrofolate Reductase Inhibitors Targeting Trimethoprim Resistant Enzymes Identified in Clinical Isolates of Methicillin Resistant Staphylococcus aureus. ACS Infectious Diseases 2019, 5, 1896-1906, https://dx.doi.org/10.1021\%2Facsinfecdis.9b00222.

10. Estrada, A.; Wright, D.L.; Anderson, A.C. Antibacterial Antifolates: From Development through Resistance to the Next Generation. Cold Spring Harb Perspect Med 2016, 6, https://doi.org/10.1101/cshperspect.a028324.

11. Lowy, F.D. Antimicrobial resistance: the example of Staphylococcus aureus. The Journal of Clinical Investigation 2003, 111, 1265-1273, https://dx.doi.org/10.1172\%2FJCI200318535.

12. Nurjadi, D.; Olalekan, A.O.; Layer, F.; Shittu, A.O.; Alabi, A.; Ghebremedhin, B.; Schaumburg, F.; Hofmann-Eifler, J.; Van Genderen, P.J.J.; Caumes, E.; Fleck, R.; Mockenhaupt, F.P.; Herrmann, M.; Kern, W.V.; Abdulla, S.; Grobusch, M.P.; Kremsner, P.G.; Wolz, C.; Zanger, P. Emergence of trimethoprim resistance gene dfrG in Staphylococcus aureus causing human infection and colonization in sub-Saharan Africa and its import to Europe. Journal of Antimicrobial Chemotherapy 2014, 69, 2361-2368, https://doi.org/10.1093/jac/dku174.

13. Hawser, S.; Lociuro, S.; Islam, K. Dihydrofolate reductase inhibitors as antibacterial agents. Biochemical Pharmacology 2006, 71, 941-948, https://doi.org/10.1016/j.bcp.2005.10.052.

14. Banerjee, M.; Parai, D.; Chattopadhyay, S.; Mukherjee, S.K. Andrographolide: antibacterial activity against common bacteria of human health concern and possible mechanism of action. Folia Microbiologica 2017, 62, 237-244, https://doi.org/10.1007/s12223-017-0496-9.

15. Saranya, S.; Aswini, M.; Sangeetha, M.K. Molecular interaction of phytochemicals with snake venom: Phytochemicals of Andrographis paniculata inhibits phospholipase A2 of Russell's viper (Daboia russelli). Biocatal. Agric. Biotechnol 2019, 18, https://doi.org/10.1016/j.bcab.2019.101058

16. Morris, G.M.; Goodsell, D.S.; Halliday, R.S.; Huey, R.; Hart, W.E.; Belew, R.K.; Olson, A.J. Automated docking using a Lamarckian genetic algorithm and an empirical binding free energy function. Journal of Computational Chemistry 1998, 19, 1639-1662, https://doi.org/10.1002/(SICI)1096987X(19981115)19:14<1639::AID-JCC10>3.0.CO;2-B.

17. Van Boeckel, T.P.; Gandra, S.; Ashok, A.; Caudron, Q.; Grenfell, B.T.; Levin, S.A.; Laxminarayan, R. Global antibiotic consumption 2000 to 2010: an analysis of national pharmaceutical sales data. The Lancet Infectious Diseases 2014, 14, 742-750, https://doi.org/10.1016/S1473-3099(14)70780-7.

18. McGettigan, P.; Roderick, P.; Kadam, A.; Pollock, A. Threats to global antimicrobial resistance control: Centrally approved and unapproved antibiotic formulations sold in India. British Journal of Clinical Pharmacology 2019, 85, 59-70, https://doi.org/10.1111/bcp.13503.

19. Lynch, G.; Magill, G.B.; Sordillo, P.; Golbey, R.B. Combination chemotherapy of advanced sarcomas in adults with "CYOMAD" (S7). Cancer 1982, 50, 1724-1727, https://doi.org/10.1002/10970142(19821101)50:9\%3C1724::aid-cncr2820500912\%3E3.0.co;2-1.

20. Mennes, M.; Stiers, P.; Vandenbussche, E.; Vercruysse, G.; Uyttebroeck, A.; Meyer, G.D.; Van Gool, S.W. Attention and information processing in survivors of childhood acute lymphoblastic leukemia treated with chemotherapy only. Pediatric Blood \& Cancer 2005, 44, 479-486, https://doi.org/10.1002/pbc.20147.

21. Daw, N.C.; Billups, C.A.; Rodriguez-Galindo, C.; McCarville, M.B.; Rao, B.N.; Cain, A.M.; Jenkins, J.J.; Neel, M.D.; Meyer, W.H. Metastatic osteosarcoma. Cancer 2006, 106, 403-412, https://doi.org/10.1002/cncr.21626.

22. Bell, D.J.; Nyirongo, S.K.; Mukaka, M.; Zijlstra, E.E.; Plowe, C.V.; Molyneux, M.E.; Ward, S.A.; Winstanley, P.A. Sulfadoxine-Pyrimethamine-Based Combinations for Malaria: A Randomised Blinded 
Trial to Compare Efficacy, Safety and Selection of Resistance in Malawi. PLOS ONE 2008, 3, https://doi.org/10.1371/journal.pone.0001578.

23. Zhanel, G.G.; DeCorby, M.; Laing, N.; Weshnoweski, B.; Vashisht, R.; Tailor, F.; Nichol, K.A.; Wierzbowski, A.; Baudry, P.J.; Karlowsky, J.A.; Lagacé-Wiens, P.; Walkty, A.; McCracken, M.; Mulvey, M.R.; Johnson, J.; Hoban, D.J. Antimicrobial-Resistant Pathogens in Intensive Care Units in Canada: Results of the Canadian National Intensive Care Unit (CAN-ICU) Study, 2005-2006. Antimicrobial Agents and Chemotherapy 2008, 52, 1430-7, https://doi.org/10.1128/AAC.01538-07.

24. Muddala, N.P.; White, J.C.; Nammalwar, B.; Pratt, I.; Thomas, L.M.; Bunce, R.A.; Berlin, K.D.; Bourne, C.R. Inhibitor design to target a unique feature in the folate pocket of Staphylococcus aureus dihydrofolate reductase. European Journal of Medicinal Chemistry 200, https://doi.org/10.1016/j.ejmech.2020.112412.

25. Margosiak, S.A.; Appleman, J.R.; Santi, D.V.; Blakley, R.L. Dihydrofolate Reductase from the Pathogenic Fungus Pneumocystis carinii: Catalytic Properties and Interaction with Antifolates. Archives of Biochemistry and Biophysics 1993, 305, 499-508, https://doi.org/10.1006/abbi.1993.1453.

26. Dale, G.E.; Broger, C.; D' Arcy, A.; Hartman, P.G.; DeHoogt, R.; Jolidon, S.; Kompis, I.; Labhardt, A.M.; Langen, H.; Locher, H.; Page, M.G.P.; Stüber, D.; Then, R.L.; Wipf, B.; Oefner, C. A single amino acid substitution in Staphylococcus aureus dihydrofolate reductase determines trimethoprim resistance. Journal of Molecular Biology 1997, 266, 23-30, https://doi.org/10.1006/jmbi.1996.0770.

27. Wróbel, A.; Arciszewska, K.; Maliszewski, D.; Drozdowska, D. Trimethoprim and other nonclassical antifolates an excellent template for searching modifications of dihydrofolate reductase enzyme inhibitors. The Journal of Antibiotics 2020, 73, 5-27, https://doi.org/10.1038/s41429-019-0240-6.

28. Veloso, D.J.; Abrão, F.; Martins, C.H.G.; Bronzato, J.D.; Gomes, B.P.F.A.; Higino, J.S.; Sampaio, F.C. Potential antibacterial and anti-halitosis activity of medicinal plants against oral bacteria. Archives of Oral Biology 2020, 110, https://doi.org/10.1016/j.archoralbio.2019.104585.

29. Soleymani, S.; Farzaei, M.H.; Zargaran, A.; Niknam, S.; Rahimi, R. Promising plant-derived secondary metabolites for treatment of acne vulgaris: a mechanistic review. Archives of Dermatological Research 2020, 312, 5-23, https://doi.org/10.1007/s00403-019-01968-z.

30. Mohotti, S.; Rajendran, S.; Muhammad, T.; Strömstedt, A.A.; Adhikari, A.; Burman, R.; de Silva, E.D.; Göransson, U.; Hettiarachchi, C.M.; Gunasekera, S. Screening for bioactive secondary metabolites in Sri Lankan medicinal plants by microfractionation and targeted isolation of antimicrobial flavonoids from Derris scandens. Journal of Ethnopharmacology 2020, 246, https://doi.org/10.1016/j.jep.2019.112158.

31. Cordeiro, L.; Figueiredo, P.; Souza, H.; Sousa, A.; Andrade-Júnior, F.; Barbosa-Filho, J.; Lima, E. Antibacterial and Antibiofilm Activity of Myrtenol against Staphylococcus aureus. Pharmaceuticals 2020, 13, https://doi.org/10.3390/ph13060133.

32. Wang, L.; Huang, Y.; Yin, G.; Wang, J.; Wang, P.; Chen, Z.-Y.; Wang, T.; Ren, G. Antimicrobial activities of Asian ginseng, American ginseng, and notoginseng. Phytotherapy Research 2020, 34, 1226-1236, https://doi.org/10.1002/ptr.6605.

33. Revee, S.M.; Gainza, P.; Frey, K.M.; Georgiev, I.; Donald, B.R.; Anderson, A.C. Protein design algorithm predict viable resistance to an experimental antifolates. J Proceedings of the National Academy of Sciences 2015, 112, 749-754, https://doi.org/10.1073/pnas.1411548112.

34. Bhosle, A.; Chandra, N. Structural analysis of dihydrofolate reductases enables rationalization of antifolate binding affinities and suggests repurposing possibilities. The FEBS Journal 2016, 283, 1139-1167, https://doi.org/10.1111/febs.13662.

35. Moon, A.; Khan, D.; Gajhbiye, P.; Jariya, M. In silico studies of inhibitors of dihydrofolate reductase and dihydropterate synthase of E. coli. International Journal of Pharmacy and Technology 2017, 9, 2881628829.

36. Majumdar, M.; Misra, T.K.; Roy, D.N. In vitro anti-biofilm activity of 14-deoxy-11,12didehydroandrographolide from Andrographis paniculata against Pseudomonas aeruginosa. Brazilian Journal of Microbiology 2020, 51, 15-27, https://doi.org/10.1007/s42770-019-00169-0.

37. Zhang, L.; Bao, M.; Liu, B.; Zhao, H.; Zhang, Y.; Ji, X.; Zhao, N.; Zhang, C.; He, X.; Yi, J.; Tan, Y.; Li, L.; Lu, C. Effect of Andrographolide and Its Analogs on Bacterial Infection: A Review. Pharmacology 2020, 105, 123-134, https://doi.org/10.1159/000503410.

38. Kumar, G.; Singh, D.; Tali, J.A.; Dheer, D.; Shankar, R. Andrographolide: Chemical modification and its effect on biological activities. Bioorganic Chemistry 2020, https://doi.org/10.1016/j.bioorg.2019.103511.

39. Ali, S.; Mir, S.A. Antibacterial activity of Andrographis paniculata of methanolic extract against some human pathogenic bacteria and effect of andrographolide compound against bacterial pathogen. Int $J$ Pharmaceut Sci Res 2020, 11, 1146-1151, http://doi.org/10.13040/IJPSR.0975-8232.11(3).1146-51.

40. Mishra, U.S.; Mishra, A.; Kumari, R.; Murthy, P.N.; Naik, B.S. Antibacterial activity of ethanol extract of Andrographis paniculata. Indian J Pharm Sci 2009, 71, 436-438.

41. Malahubban, M.; Alimon, A.R.; Sazili, A.Q.; Fakurazi, S.; Zakry, F.A. Phytochemical analysis of Andrographis paniculata and Orthosiphon stamineus leaf extracts for their antibacterial and antioxidant potential. Tropical biomedicine 2013, 30, 467-480. 
42. Adaramola, B.; Benjamin, G.; Oluchi, O.; Fapohunda, S. Antimicrobial and antioxidant activities of crude methanol extract and fractions of Andrographis paniculata leaf (Family: Acanthaceae) (Burm. f.) wall. Ex Nees. Jordan Journal of Biological Sciences 2018, 11, 23-30.

43. Premanath, R.; Devi, N.L. Antibacterial, antifungal and antioxidant activities of Andrographis paniculata Nees. Leaves. Int J Pharmaceut Sci Res 2011, 2091-2099, http://dx.doi.org/10.13040/IJPSR.09758232.2(8).2091-99.

44. Shalini, V.B.; Narayanan, J.S. ntibacterial activity of Andrographis paniculata Nees against selective human pathogens. African Journal of Microbiology Research 2015, 9, 1122-1127, https://doi.org/10.5897/AJMR2015.7515.

45. Sule, A.; Uddin, Q.; Samah, O.; Omar, M. Screening for Antibacterial Activity of Andrographis paniculata Used in Malaysian Folkloric Medicine: A Possible Alternative for the Treatment of Skin Infections. Ethnobotanical leaflets 2010, 14, 445-456.

46. Aabraham, A.O.; Nasiru, A.U.; Abdulazeez, A.K.; Seun, O.O.; Ogonna, D.W. Therapeutic potency of the polar and non-polar extracts of Andrographis paniculata leaf against some pathogenic bacterial isolates. Arch Pharmacy Pharmacol Res 2019.

47. Chauhan, E.S.; Sharma, K.; Bist, R. Andrographis paniculata: A Review of its Phytochemistry and Pharmacological Activities. Res J Pharmacy Tech 2019, 12, 891-900, https://doi.org/10.5958/0974360X.2019.00153.7.

48. Pandey, J.; Saini, V.; Tiwari, S.; Raja, W. A Study of Antibacterial Activity of Andrographis Paniculata Leaf And Stem Bark Extracts Against Some Clinical Pathogenic Bacteria's. Asian Journal of Pharmaceutical and Biological Research 2019, 7, 65-70. 